\title{
A short-term yoga-based intervention improves balance control, body composition, and some aspects of mental health in the elderly men
}

\author{
Milada Krejčí ${ }^{1}$, Rudolf Psotta ${ }^{2, *}$, Martin Hill ${ }^{3}$, Jiří Kajzar ${ }^{1}$, Dobroslava Jandová1, and Václav Hošek ${ }^{1}$ \\ ${ }^{I}$ College of Physical Education and Sport PALESTRA, Prague, Czech Republic; ${ }^{2}$ Faculty of Physical Culture, Palacký Uni- \\ versity Olomouc, Olomouc, Czech Republic; and ${ }^{3}$ Institute of Endocrinology, Prague, Czech Republic
}

Copyright: (C) 2020 M. Krejčí et al. This is an open access article licensed under the Creative Commons Attribution License (https://creativecommons.org/licenses/by/4.0/).

\begin{abstract}
Background: Body balance control represents a key function for the physical activities of elderly people aged sixty-five and over. Due to the biological and psychosocial changes in this age group, yoga-based intervention appears to be beneficial to maintaining their ability to balance along with related mental aspects of health. Objective: The aim of the presented study was to examine the effects of the 4-week yoga-based intervention on balance, body composition and the mental health aspects of elderly men. Methods: 67-80-year-old elderly men $(N=234$, mean age $72.5 \pm 7.7$ years) were assessed with The Tinetti Balance Assessment Tool, bioimpedance body composition analyser InBody 230 and the Health Survey SF-36, applied for the pre- and post-testing. The experimental group $(n=122)$ underwent the 4-week intervention based on yoga exercises, while the control group $(n=112)$ had a usual daily program at their senior homes or centers. Results: The yoga intervention led to significant improvement of both the static balance and gait scores compared to the control group, with medium effect size, $\eta_{\mathrm{p}}^{2}=.070$ and .080 . The intervention also had an effect on the decrease of body fat percentage (by 1.7\%) and an increase in muscle mass (by $1.3 \mathrm{~kg}$ ), with a large effect size, $\eta_{\mathrm{p}}^{2}=.214$ and .301 . Results of the Health Survey showed that overall mental health did not change significantly although positive improvement in two items connected with emotional problems. Conclusions: A short-term yogabased intervention may have a significant positive influence on physical abilities such as balance control and body composition rather than on mental health aspects in elderly men aged sixty-five and over.
\end{abstract}

Keywords: exercise, functional mobility, psychic state, seniors

\section{Introduction}

With increasing age, postural control and balance skills begin to worsen (Buso et al., 2019; Shumway-Cook \& Woollacott, 2016). Impaired balance may result in a decrease in physical activity during daily life and concurrently represents a major risk factor for older people when it comes to falling (Bukova et al., 2019). Fear of falling and the consequent loss of independence are also common problems among the elderly (Dellinger, 2017). Reduction in the deterioration of posture control is therefore considered a key factor in the prevention of falls and subsequent injuries. Incidental falls of the elderly rank high in the category of geriatric health problems that have negative consequences

\footnotetext{
* Address for correspondence: Rudolf Psotta, Department of Natural Sciences in Kinanthropology, Faculty of Physical Culture, Palacký University Olomouc, tř. Míru 117, 77111 Olomouc, Czech Republic. E-mail: rudolf.psotta@upol.cz
}

on the physical, mental and social quality of life of elderly people over 65 years (Ni Chronin, Ni Chronin, $\&$ Beveridge, 2015). Additionally, these problems also have a high cost of treatment. Since 2000 in the Czech Republic, similarly as in other European countries, the number of persons aged 65 and over has been continuously increasing and as a consequence, treatment costs have become higher.

Motor performance and learning issues are specific in the elderly due to the potential dysfunction of the central and peripheral nervous system as well as the neuromuscular system. These specifics manifest themselves as coordination difficulties, lack of fitness, slower and less precise movements, balance and gait difficulties (Gilleard \& Higgs, 2005). These limits may have a negative effect on the ability of the elderly to carry out routine daily activities, including self-care activities. Balance problems during walking with the consequence of a fall are the main source of injury and morbidity in the elderly: $20-30 \%$ of the elderly who 
suffer from a fall suffer moderate to severe injuries that limit mobility and reduce the quality of life (Hafström, Malmström, Terdèn, Fransson, \& Magnusson, 2016). Slower information processing can also affect motor performance in the elderly (Gillespie et al., 2012). The findings of Bendíková (2017) show that amongst the elderly, movement activities alone play an important role in maintaining social contacts, health and independence, the values of which are particularly specific for the life of the elderly from different points of view.

Recent reviews of literature show that the practicing of yoga exercises may bring advantages to various groups of the elderly, including mental health, psychosocial functioning (e.g., Butterfield, Schultz, Rasmussen, \& Proeve, 2017; Pascoe \& Bauer, 2015), physical functioning and motor functions (Wang, 2009; Youkhana, Dean, Wolff, Sherrington, \& Tiedemann, 2016). These authors pointed out the positive effects of yoga interventions in decreasing depression, mood, stress, fear, anxiety, sleep disorders, and on the enhancement of concentration, emotional stability, self-confidence, social skills and social engagement. The positive benefits of yoga programs were proved in healthy elders as well as in older individuals with cancer, multiple sclerosis, diabetes, chronic pancreatitis, asthma and fibromyalgia (Oken et al., 2006; Tew, Howsam, Hardy, \& Bissell, 2017). For the maintenance of posture function and balance abilities in the elderly, yoga exercises may provide a wide range of different movement situations that combine the physical and psychosocial attributes of balance, such as synchronization of movement with breathing, slowed movement sequences, and releasing the mind from fear and worry (Krejci \& Kornatovska, 2017; Maheshwarananda, 2000).

However, the previous yoga-based intervention studies with the elderly included women only or gender-mixed samples in which the number of women was very often considerably higher than men. Importantly, the possible effects of yoga were not analyzed separately for elder men and women in those studies. That is in contradiction to possible sex differences and the effects of yoga exercises on these differences, due to the biological and psychosocial specifics of older men and women. The studies with gender-mix samples of the elderly showed that yoga programs can enhance balance and other motor functions that are associated with balance such as muscle strength, flexibility and mobility (e.g., Oken et al., 2006; Tiedemann, O’Rourke, Sesto, \& Sherrington, 2013). As a consequence of these effects, fear of falling may decrease (Schmid, Van Puymbroeck, \& Koceja, 2010).

Effects of yoga programs on mental health were also demonstrated on common samples of elderly men and women, such as a decrease in depression, social dysfunction (Ebrahimi, Esmaeilzadeh, \& Mohamad, 2019), stress levels (Lindahl, Tilton, Eickholt, \& Ferguson-Stegall, 2016), an additional stimulation of a sense of well-being and energy (Oken et al., 2006), an increase in self-esteem and a positive attitude towards the surrounding world (Krejci, 2011).

However, to the authors' knowledge, no intervention study has been published that would examine the effects of yoga on the balance and mental functionality specifically in elderly men. A focus of this research on the effects of yoga on balance shows it to be relevant when poorer balance was revealed as one of the significant risk factors of incident falls in elderly men but not in women (Gale, Cooper, \& Aihie Sayer, 2016; Gale, Westbury, Cooper, \& Dennison, 2018). In addition, it is also important to note that the possible effects of yoga on the mental and physical health of elders were examined mostly in those practicing this activity for longer periods of time, eight weeks or more. Therefore, the aim of the presented study was to examine the effects of short-term yoga intervention on balance, body composition, and the mental health aspects in elderly men. On the basis of the knowledge introduced above on the possible effects of yoga exercises, we hypothesized that short-term intensive yoga intervention in elderly men may lead to the optimization of balance abilities and aspects of physical, mental, emotional and social functioning.

\section{Methods}

\section{Participants}

The elderly men $(N=234)$, mean age $72.5 \pm 7.7$ years (range 67-80 years) were recruited from senior homes and centers on the base of voluntary participation. The participants were randomly assigned to the experimental (yoga) group (Exp; $n=122$ ), mean age $72.8 \pm 7.4$ years, body height $173.4 \pm 7.9 \mathrm{~cm}$; or the control group (Con; $n=112$ ), mean age $72.2 \pm 6.5$ years, body height $174.0 \pm 6.6 \mathrm{~cm}$. Results of the independent $t$-test $(\alpha=.05)$ showed that the groups did not differ significantly in age $(p=.215)$, body height $(p=.684)$, body weight $(p=.689)$ and body mass index (BMI; $p=.445$ ) at the beginning of the experiment. While randomizing, equal or very similar number of participants for both groups were kept in the particular homes/centers. The exclusion criteria for the involvement of participants in the experimental study has been determined according to the White Book on Physical and Rehabilitation Medicine in Europe (Gutenbrunner, Ward, \& Chamberlein, 2007) and are as follow: (i) human to human infectious diseases and bacillus carrier, (ii) all acute-stage diseases 
and conditions in which destabilization of health state can be reasonably expected, (iii) cachexia of various etiologies, (iv) malignant tumors, (v) active attacks or phases of psychoses and mental disorders with asocial manifestations or with reduced communication, (vi) 2nd and 3rd degree of urinary incontinence and stool incontinence.

Written informed consent to participation in the study was obtained from the participants. Participants of the control group were informed about the physical, motor and health status assessment, while participants of the experimental group were provided with the information on both the diagnostic and intervention parts of the program. However, participants of both groups were not informed about the research purpose of the study. The Ethical committee of the research institution expressed full agreement with the research aims and procedures. Research conformed to the requirements stipulated in the Declaration of Helsinki.

\section{Instruments of assessment}

\section{Medical anamnesis}

The standardized medical anamnesis protocol was applied to assess the health state, drug consumption, disabilities, operations, etc. of each participant, and finally, the physician carried out the medical recommendation/ decision to include an individual in the study.

\section{Assessment of body composition}

Body height was measured using the Tanita Leicester Height Measure device (Invicta Plastics, Leicester, United Kingdom) with an accuracy of $0.1 \mathrm{~cm}$. A tetrapolar multi-frequency bioelectrical impedance device InBody 230 (InBody, Seoul, South Korea) was used to assess body weight, BMI, body fat percentage and total muscle mass.

\section{Balance assessment}

The Tinetti Balance Assessment Tool is a clinical performance-oriented exam used to measure the static and dynamic balance in older adults (Tinetti, Williams, \& Mayewski, 1986). The static balance test is assessed on the basis of observation of an individual's bodily behavior during sitting and standing positions and their changes which are defined by nine items. Dynamic balance is judged by eight items that involve characteristics of gait manifested in an individual during a walk across a $4.5-\mathrm{m}$ walkway, first at their usual pace, then at a rapid pace. The particular items of both static and dynamic balance are rated on the 2-point scale or the 3-point scale. The maximal total scores are 16 and 12 points for static balance and dynamic balance (gait), respectively. The higher the score, the better the performance.
The Tinetti Balance Assessment Tool, also named the Tinetti Mobility Test reported good to excellent intra- and inter-rater reliability, intraclass correlation coefficient $>.80$, and medium sensitivity and specificity to identify fallers (Kegelmeyer, Kloos, Thomas, \& Kostyk, 2007).

\section{RAND 36 Short Form Health Survey (SF-36)}

This survey (SF-36) is a valid and reliable indicator of overall health status (Ware, Snow, Kosinski, \& Gandek, 1993), and it is widely used all over the world today. It is comprised of 36 items that assess eight categories: physical functioning (10 items), physical role of functioning (4 items), emotional role functioning ( 3 items), social role functioning ( 2 items), mental health (5 items), vitality (4 items), body pain ( 2 items), and general health perceptions ( 5 items). Responses across the items are scored on the two- to six-point scales. One item (question 2) covers a change in health status over the past year. Therefore, participants in the presented study were not assessed on this item. The method was reported to have a reliability of $r=.65$ to .94 across the scales, with a median of .85 . This resulted in very good internal consistency and item-discriminant validity (McHorney, Ware, Lu, \& Sherbourne, 1994).

\section{Procedures}

The investigations started with the medical anamnesis provided by a physician, followed by a bioimpedance assessment of body composition provided by an anthropologist with two assistants. After the two medical assessments, a physiotherapist conducted an individual balance exam using the Tinetti Balance Assessment Tool, according to the protocol by the Washington Health Care Association (2013).

Then, each participant completed the SF-36 survey with the assistance of a research team member. Each participant was instructed on how to respond to the items on the survey and was told to relax and answer truthfully. No time limit was stated for them to fulfill the survey. The survey was used according to its official manual and interpretation guide (Ware et al., 1993). Each item of the SF-36 was analyzed as to their possible intervention effect. Each participant underwent all of the procedures in a single go. Obtained data was stored and protected according to the Regulation of the European Parliament and the Council of the EU 2016/679. After the pre-measurements, participants of the experimental group underwent the 4-week yogabased intervention, while the control group ran the standard daily routine program in their senior homes or centers. One to six days after the 4-week intervention, the post-measurements were performed in the same conditions. 


\section{Yoga-based intervention}

The 4-week intervention of the experimental group was focused on body posture and balance control, flexibility, muscle strength, breathing, the stimulation of psychic harmony and the optimization of social interaction. These yoga exercises were carried out in accordance with the system of Yoga in Daily Life (Maheshwarananda, 2000), and without contraindications to the elderly (Sarvahita Asanas), and the movements whilst sitting on a chair or standing. Once per week, the main training lesson lasting 90 minutes was conducted with groups of 10-12 participants under the guidance of the coach, and with two or three coach assistants who helped seniors to complete exercises easily and correctly during each lesson. After each main training, each participant received an educational sheet, which contained a simple guide and a symbolic attribute for the concrete intervention week. During the week, participants repeated practiced exercises and yoga elements each day in 5-10-minute intervals. The assistants also motivated participants during these weeks to repeat learned exercises. The week program also included the motto: Week 1 "You are never alone", Week 2 "Change is always possible", Week 3 "Movement is life", Week 4 "Enjoy life and every moment" (Krejčí, 2019). During the intervention, the control group had their usual daily regiment at their senior homes or centers.

\section{Statistical analysis}

Respecting the skewed distribution and non-constant variance in most dependent variables, these were transformed by Box-Cox transformations to achieve data symmetry and homoscedasticity prior to further processing. The homogeneity and distribution of the transformed data and residuals were checked by residual analysis as described elsewhere (Meloun et al., 2004).

The analysis of variance (ANOVA) model was used for the evaluation of the relationships between dependent variables on one side and the experimental group, measurement and individual subjects on the other side. Therefore, the model consisted of Subject factor explaining inter-individual variability between subject factor Group (control × experimental group) and within subject factors Measurement (before intervention $\times$ after intervention), and significant Group $\times$ Measurement interaction indicating whether the Group factor significantly influences the change after intervention). Less significant multiple comparisons followed the ANOVA testing. The results obtained from the transformed data (subgroup means and their 95\% confidence intervals) were re-transformed to the original scale for the presentation. The effect size of the factors and the Group $\times$ Measurement interaction was calculated using partial eta squared $\left(\eta_{\mathrm{p}}^{2}\right)$ with an interpretation of $\eta_{\mathrm{p}}^{2}=.01, .06$, and .14 as small, medium, and large effects, respectively. A significance level of $\alpha=.05$ was set for all tests. Statistical software Statgraphics Centurion (Version 18; Statgraphic Technologies, The Plains, VA, USA) was used for the statistical analysis.

\section{Results}

\section{Intervention effects on body composition}

The intervention led to the significant improvement of two measurements of body composition. Firstly, the Exp group decreased in body fat percentage by $1.7 \%$ of fat while the Con group increased the median of this variable by $0.3 \%$ of body fat (Table 1 ). The analysis showed the effect of the Measurement on body fat percentage and Group $\times$ Measurement interaction was statistically significant, $p<.001$, with a large effect size, $\eta_{\mathrm{p}}^{2}=.214$ (Table 1). Second measurements revealed that total muscle mass increased by $1.3 \mathrm{~kg}$ (median) in the Exp group after the yoga intervention, while there was no change in the median of muscle mass in the Con group. The analysis showed the effect of both the Measurement and Group $\times$ Measurement (both $p<.001)$, with a large effect size of the interaction $\eta_{\mathrm{p}}^{2}=.301($ Table 1$)$.

The effect of Measurement was also significant for BMI $(p=.02)$ with a decrease in both groups, however, the Group $\times$ Measurement interaction was not significant ( $p=.38$; Table 1). Concurrently, the effects of Measurement and Group $\times$ Measurement interaction were not significant for body weight.

\section{Intervention effects on balance}

The effect of Measurement was significant for performance in both Tinetti static and dynamic balance test, $p<.001$ and $p=.010$, respectively. The significant Group $\times$ Measurement interaction $(p<.001)$ showed that the improvement was significantly higher for static and dynamic balance in the Exp group, from 13.2 to 13.8 and 9.9 to 10.2 , respectively, as compared to the Con group, from 13.8 to 13.9 and 10.4 to 10.3 , respectively, both with medium effect, $\eta_{\mathrm{p}}^{2}=.070$ and .080 , respectively (Table 2 ). The positive effect of the yoga-based intervention was also indicated by the significance of Measurement and Group $\times$ Measurement interaction for the total score of the Tinetti balance test (both $p<.001$ ) and medium effect size of the interaction, $\eta_{\mathrm{p}}^{2}=.108$ (Table 2).

\section{Intervention effects on the health and quality of life mea- sures of the SF-36 Survey}

The effect of the intervention was found to improve the self-care abilities item (PF10) of the Physical 
Table 1

Body composition measures in the pre- and post-intervention

\begin{tabular}{|c|c|c|c|c|c|c|}
\hline \multirow[b]{2}{*}{ Measure } & \multicolumn{2}{|c|}{ Measurement } & \multicolumn{3}{|c|}{$p$} & \multirow{2}{*}{$\begin{array}{c}\eta_{\mathrm{p}}^{2} \text { Group } \times \\
\text { Pre-Post }\end{array}$} \\
\hline & Pre & Post & Group & Pre-Post & Group $\times$ Pre-Post & \\
\hline \multicolumn{7}{|c|}{ Body weight (kg) } \\
\hline Con & $83.6[83.5,83.8]$ & $83.4[83.3,83.5]$ & & & & \\
\hline Exp & $84.3[84.2,84.4]$ & $84.3[84.2,84.4]$ & $<.001$ & .216 & .232 & .009 \\
\hline \multicolumn{7}{|c|}{ Body mass index $\left(\mathrm{kg} / \mathrm{m}^{2}\right)$} \\
\hline Con & $27.5[27.4,27.5]$ & $27.4[27.3,27.4]$ & & & & \\
\hline Exp & $27.7[27.7,27.8]$ & $27.7[27.6,27.7]$ & $<.001$ & .017 & .384 & .005 \\
\hline \multicolumn{7}{|c|}{ Body fat (\%) } \\
\hline Con & $26.6[26.3,26.9]$ & $26.9[26.6,27.2]$ & & & & \\
\hline Exp & $26.9[26.7,27.2]$ & $25.2[25.0,25.5]$ & $<.001$ & $<.001$ & $<.001$ & .214 \\
\hline \multicolumn{7}{|c|}{ Muscle mass (kg) } \\
\hline Con & $33.9[33.8,34.1]$ & $33.9[33.7,34.0]$ & & & & \\
\hline Exp & $34.8[34.7,34.9]$ & $36.1[36.0,36.2]$ & $<.001$ & $<.001$ & $<.001$ & .301 \\
\hline
\end{tabular}

Note. $\quad$ Con = control group; Exp = experimental group. Scores are presented as median [95\% confidence interval of median] .

Table 2

The Tinetti balance test scores in the pre- and post-intervention

\begin{tabular}{|c|c|c|c|c|c|c|}
\hline \multirow[b]{2}{*}{ Measure } & \multicolumn{2}{|c|}{ Measurement } & \multicolumn{3}{|c|}{$p$} & \multirow{2}{*}{$\begin{array}{c}\eta_{\mathrm{p}}^{2} \text { Group } \times \\
\text { Pre-Post }\end{array}$} \\
\hline & Pre & Post & Group & Pre-Post & Group $\times$ Pre-Post & \\
\hline \multicolumn{7}{|c|}{ Static balance } \\
\hline Con & $13.8[13.7,13.9]$ & $13.9[13.8,14.0]$ & & & & \\
\hline Exp & $13.2[13.2,13.3]$ & $13.8[13.7,13.8]$ & $<.001$ & $<.001$ & $<.001$ & .070 \\
\hline \multicolumn{7}{|c|}{ Dynamic balance (gait score) } \\
\hline Con & $10.4[10.3,10.5]$ & $10.3[10.3,10.4]$ & & & & \\
\hline Exp & $9.9[9.8,10.0]$ & $10.2[10.2,10.3]$ & $<.001$ & .010 & $<.001$ & .080 \\
\hline \multicolumn{7}{|c|}{ Total score of balance } \\
\hline Con & $24.1[24.0,24.3]$ & $24.2[24.1,24.3]$ & & & & \\
\hline Exp & $23.1[23.0,23.2]$ & $23.9[23.8,24.0]$ & $<.001$ & $<.001$ & $<.001$ & .108 \\
\hline
\end{tabular}

Note. $\quad$ Con = control group; Exp = experimental group. Scores are presented as median [ $95 \%$ confidence interval of median].

functioning (PF) category. The effect of the Measurement was marginal, $p=.06$, however Group $\times$ Measurement interaction was significant for the PF10 item, $p=.01$ (Table 3).

The effects of Measurement and Group $\times$ Measurement interaction were not significant for the RP1, RP2 and RP3 items of the Role Limitations due to physical health problems (RP) category (Table 3). Although both groups improved the score in the RP4 item, reflected in a significant effect of Measurement $p=.02$, Group $\times$ Measurement interaction was not significant, $p=.18$ (Table 3).

The Con group improved the score of the state of calmness and peacefulness indicated by the MH3 item of the Mental health $(\mathrm{MH})$ category in a larger extent, with the significant interaction $p=.02$ (Table 4). Furthermore, the Con group improved significantly its state of happiness, the MH5 item, while worsening the score in the Exp group, the interaction Group $\times$ Measurement $p=.01$ (Table 4).

The yoga-based intervention led to significant positive changes in two of three items related to the Role limitations due to emotional problems (RE) category, with significant Group $\times$ Measurement interaction, $p=.03$. Secondly, the Exp group improved the score of the RE3 item while there were no changes in the median score of the Con group. The effects of both Measurement and Group $\times$ Measurement interaction on the RE3 item score were significant, both $p=.03$ (Table 4). 
Table 3

Physical functioning, Role limitations due to physical health problems and Bodily pain measures of the RAND 36 Short Form Health Survey in the pre- and post-intervention

\begin{tabular}{|c|c|c|c|c|c|c|}
\hline \multirow[b]{2}{*}{ Scale (score); Item } & \multicolumn{2}{|c|}{ Measurement } & \multicolumn{3}{|c|}{$p$} & \multirow{2}{*}{$\begin{array}{c}\eta_{\mathrm{p}}^{2} \text { Group } \times \\
\text { Pre-Post }\end{array}$} \\
\hline & Pre & Post & Group & Pre-Post & Group $\times$ Pre-Post & \\
\hline
\end{tabular}

PF1 (1-3+); Health limit: vigorous activities

$\begin{array}{lll}\text { Con } & 1.77[1.74,1.80] & 1.72[1.69,1.75] \\ \text { Exp } & 1.47[1.44,1.49] & 1.49[1.46,1.51]\end{array}$

PF2 (1-3+); Health limit: moderate activities

$\begin{array}{lll}\text { Con } & 2.62[2.59,2.65] & 2.62[2.59,2.65] \\ \text { Exp } & 2.29[2.26,2.31] & 2.34[2.31,2.36]\end{array}$

$$
<.001
$$

PF3 (1-3+); Health limit: lifting/carrying groceries

$$
\begin{array}{lll}
\text { Con } & 2.80[2.78,2.83] & 2.78[2.75,2.80] \\
\text { Exp } & 2.65[2.63,2.67] & 2.68[2.66,2.70]
\end{array}
$$

PF4 (1-3+); Health limit: climbing of several flights/stairs

$$
\begin{array}{lll}
\text { Con } & 2.44[2.41,2.46] & 2.42[2.39,2.44] \\
\text { Exp } & 2.09[2.06,2.11] & 2.13[2.11,2.15]
\end{array}
$$

PF5 (1-3+); Health limit: climbing of one flight/stairs

$$
\begin{array}{lll}
\text { Con } & 2.76[2.72,2.79] & 2.72[2.69,2.75] \\
\operatorname{Exp} & 2.50[2.47,2.53] & 2.51[2.48,2.54]
\end{array}
$$

PF6 (1-3+); Health limit: bending, kneeling, stopping

$$
\begin{array}{lll}
\text { Con } & 2.49[2.45,2.53] & 2.50[2.46,2.54] \\
\operatorname{Exp} & 2.24[2.20,2.27] & 2.26[2.23,2.30]
\end{array}
$$

PF7 (1-3+); Health limit: walking > 1 mile

$$
\begin{array}{lll}
\text { Con } & 2.64[2.60,2.68] & 2.57[2.53,2.61] \\
\operatorname{Exp} & 2.31[2.27,2.34] & 2.32[2.29,2.36]
\end{array}
$$

$<.001 \quad .300 \quad 089$

PF8 (1-3+); Health limit: walking several blocks

$$
\begin{array}{lll}
\text { Con } & 2.80[2.79,2.81] & 2.80[2.79,2.81] \\
\text { Exp } & 2.65[2.64,2.66] & 2.66[2.65,2.66]
\end{array}
$$

PF9 (1-3+); Health limit: walking one block

$\begin{array}{lll}\text { Con } & 2.91[2.89,2.92] & 2.89[2.87,2.90] \\ \text { Exp } & 2.86[2.84,2.88] & 2.84[2.83,2.86]\end{array}$

PF10 (1-3+); Health limit: bathing, dressing

$\begin{array}{lllllll}\text { Con } & 2.96[2.95,2.98] & 2.93[2.92,2.94] & & & & \\ \text { Exp } & 2.88[2.87,2.89] & 2.89[2.88,2.90] & <.001 & .061 & .008 & .046\end{array}$

Role limitations due to physical health problems (RP)

\begin{tabular}{ccc}
\hline RP1 (1-2+); Past 4 weeks work or other activities decreasing \\
Con & $1.78[1.73,1.82]$ & $1.81[1.77,1.86]$ \\
Exp & $1.56[1.52,1.60]$ & $1.55[1.51,1.59]$
\end{tabular}

RP2 (1-2+); Past 4 weeks accomplished less than would like

$\begin{array}{lll}\text { Con } & 1.68[1.65,1.71] & 1.70[1.67,1.73] \\ \text { Exp } & 1.44[1.41,1.46] & 1.49[1.47,1.52]\end{array}$

$$
<.001
$$$$
.633
$$$$
.442
$$

RP3 (1-2+); Past 4 weeks limited in work

$$
<.001
$$

.098

.388

.005

$\begin{array}{lll}\text { Con } & 1.73[1.71,1.75] & 1.71[1.69,1.73] \\ \text { Exp } & 1.53[1.51,1.54] & 1.56[1.54,1.58]\end{array}$

$$
<.001
$$

.571

.068 
Table 3 (continued)

\begin{tabular}{|c|c|c|c|c|c|c|}
\hline \multirow[b]{2}{*}{ Scale (score); Item } & \multicolumn{2}{|c|}{ Measurement } & \multicolumn{3}{|c|}{$p$} & \multirow{2}{*}{$\begin{array}{c}\eta_{\mathrm{p}}^{2} \text { Group } \times \\
\text { Pre-Post }\end{array}$} \\
\hline & Pre & Post & Group & Pre-Post & Group $\times$ Pre-Post & \\
\hline \multicolumn{7}{|c|}{ RP4 (1-2+); Past 4 weeks difficulties in performance } \\
\hline Con & $1.72[1.69,1.74]$ & $1.73[1.71,1.76]$ & & & & \\
\hline Exp & $1.53[1.51,1.55]$ & $1.59[1.57,1.61]$ & $<.001$ & .021 & .177 & .012 \\
\hline \multicolumn{7}{|c|}{ Bodily pain (BP) } \\
\hline \multicolumn{7}{|c|}{ BP1 (+1-6); Past 4 weeks health problems interfered with normal social activities } \\
\hline Con & $2.27[2.22,2.31]$ & $2.24[2.19,2.29]$ & & & & \\
\hline Exp & $2.16[2.12,2.19]$ & $2.11[2.07,2.14]$ & $<.001$ & .183 & .687 & .001 \\
\hline \multicolumn{7}{|c|}{ BP2 (+1-5); Past 4 weeks bodily pain } \\
\hline Con & $1.74[1.68,1.80]$ & $1.70[1.64,1.76]$ & & & & \\
\hline Exp & $1.94[1.89,1.99]$ & $1.84[1.79,1.89]$ & $<.001$ & .106 & .476 & .003 \\
\hline
\end{tabular}

Note. Con = control group; Exp = experimental group. Scores are presented as median [95\% confidence interval of median].

Table 4

Social functioning, General mental health, Role limitations due to emotional problems, Vitality, energy/fatigue, and General health perceptions measures of the RAND 36 Short Form Health Survey in the pre-and post-intervention

\begin{tabular}{|c|c|c|c|c|c|c|}
\hline \multirow[b]{2}{*}{ Scale (score); Item } & \multicolumn{2}{|c|}{ Measurement } & \multicolumn{3}{|c|}{$p$} & \multirow{2}{*}{$\begin{array}{c}\eta_{\mathrm{p}}^{2} \text { Group } \times \\
\text { Measurement }\end{array}$} \\
\hline & Pre & Post & Group & Measurement & Group $\times$ Measurement & \\
\hline \multicolumn{7}{|c|}{ Social functioning (SF) } \\
\hline \multicolumn{7}{|c|}{ SF1 (+1-5); Past 4 weeks health problems interfered with normal social contacts } \\
\hline Con & $1.57[1.54,1.60]$ & $1.51[1.48,1.55]$ & & & & \\
\hline Exp & $1.77[1.74,1.80]$ & $1.77[1.74,1.80]$ & $<.001$ & .261 & .177 & .012 \\
\hline \multicolumn{7}{|c|}{ SF2 (1-5+); Past 4 weeks physical or emotional problems influenced negatively social activities } \\
\hline Con & $4.08[4.02,4.14]$ & $3.97[3.91,4.02]$ & & & & \\
\hline Exp & $4.07[4.03,4.12]$ & $4.07[4.02,4.11]$ & .197 & .105 & .141 & .014 \\
\hline \multicolumn{7}{|c|}{ General mental health $(\mathrm{MH})$} \\
\hline \multicolumn{7}{|c|}{ MH1 (1-6+); Past 4 weeks nervous } \\
\hline Con & $4.62[4.53,4.70]$ & $4.75[4.67,4.83]$ & & & & \\
\hline Exp & $4.58[4.52,4.65]$ & $4.60[4.53,4.66]$ & .080 & .168 & .248 & .009 \\
\hline \multicolumn{7}{|c|}{ MH2 (1-6+); Past 4 weeks sadness } \\
\hline Con & $5.40[5.36,5.44]$ & $5.44[5.40,5.48]$ & & & & \\
\hline Exp & $5.42[5.39,5.45]$ & $5.46[5.42,5.49]$ & .515 & .167 & .968 & $<.001$ \\
\hline \multicolumn{7}{|c|}{ MH3 (+1-6); Past 4 weeks calm, peaceful } \\
\hline Con & $2.94[2.85,3.04]$ & $2.64[2.55,2.73]$ & & & & \\
\hline Exp & $2.49[2.42,2.56]$ & $2.47[2.40,2.54]$ & $<.001$ & .008 & .018 & .036 \\
\hline \multicolumn{7}{|c|}{ MH4 (1-6+); Past 4 weeks depression } \\
\hline Con & $4.83[4.74,4.91]$ & $4.93[4.84,5.02]$ & & & & \\
\hline Exp & $4.99[4.92,5.06]$ & $5.06[5.00,5.13]$ & .008 & .113 & .775 & .001 \\
\hline \multicolumn{7}{|c|}{ MH5 (+1-6); Past 4 weeks happy } \\
\hline Con & $3.08[2.98,3.18]$ & $2.83[2.73,2.92]$ & & & & \\
\hline Exp & $2.53[2.45,2.60]$ & $2.60[2.52,2.67]$ & $<.001$ & .151 & .012 & .041 \\
\hline
\end{tabular}


Table 4 (continued)

\begin{tabular}{ccccccc}
\hline & \multicolumn{2}{c}{ Measurement } & \multicolumn{2}{c}{$p$} & $\eta_{\mathrm{p}}^{2}$ Group $\times$ \\
\cline { 2 - 3 } Scale (score); Item & Pre & Post & Group & Measurement & Group $\times$ Measurement & Measurement \\
\hline
\end{tabular}

Role limitations due to emotional problems (RE)

RE1 (1-2+); Past 4 weeks due to emotional problems cut down work or other activities

Con $\quad 1.83[1.8,1.85] \quad 1.86[1.84,1.89]$

Exp $\quad 1.77[1.75,1.78] \quad 1.77[1.76,1.79] \quad<.001 \quad .107$

.007

RE2 (1-2+); Past 4 weeks due to emotional problems accomplished less than would like

$\begin{array}{lllllll}\text { Con } & 1.85[1.83,1.87] & 1.82[1.80,1.85] & & & & \\ \operatorname{Exp} & 1.75[1.73,1.77] & 1.79[1.77,1.81] & <.001 & .624 & .030 & .030\end{array}$

RE3 (1-2+); Past 4 weeks due to emotional problems concentration decreasing
Con
$1.85[1.83,1.87] \quad 1.85[1.83,1.87]$
Exp
$1.72[1.7,1.73]$
$1.78[1.76,1.80]$
$<.001$
.032
.032
.030

Vitality, energy/fatigue (VT)

\begin{tabular}{lrl}
\hline VT1 (+1-6); Past 4 weeks full vitality & \\
Con & $2.89[2.83,2.96]$ & $2.84[2.78,2.91]$ \\
Exp & $3.03[2.98,3.09]$ & $2.93[2.87,2.98]$
\end{tabular}

VT2 (+1-6); Past 4 weeks lot of energy

$\begin{array}{lll}\text { Con } & 3.28[3.21,3.35] & 3.25[3.18,3.32] \\ \text { Exp } & 3.10[3.05,3.16] & 2.94[2.88,2.99]\end{array}$

$\operatorname{Exp}$

$3.10[3.05,3.16] \quad 2.94[2.88,2.99]$

$<.001$

.026

.134

.015

VT3 (1-6+); Past 4 weeks exhausted

$\begin{array}{lllllll}\text { Con } & 4.39[4.33,4.45] & 4.43[4.37,4.49] & & & & \\ \text { Exp } & 4.27[4.22,4.32] & 4.28[4.23,4.33] & <.001 & .493 & .696 & .001\end{array}$

VT4 (1-6+); Past 4 weeks tired

$\begin{array}{lll}\text { Con } & 3.84[3.75,3.94] & 3.97[3.88,4.06] \\ \text { Exp } & 3.82[3.75,3.90] & 3.89[3.82,3.97]\end{array}$

$.431 \quad .107$

.654

.001

General health perceptions (GH)

\begin{tabular}{|c|c|c|c|c|c|c|}
\hline \multicolumn{7}{|c|}{ GH1 (+1-5); Subjective state of health } \\
\hline Con & $3.02[2.99,3.06]$ & $3.06[3.02,3.10]$ & & & & \\
\hline Exp & $3.17[3.14,3.20]$ & $3.15[3.12,3.18]$ & $<.001$ & .800 & .218 & .010 \\
\hline \multicolumn{7}{|c|}{ GH2 (1-5+); Sick easier than others } \\
\hline Con & $3.90[3.86,3.94]$ & $3.92[3.88,3.96]$ & & & & \\
\hline Exp & $4.02[3.99,4.05]$ & $4.04[4.01,4.08]$ & $<.001$ & .393 & .943 & $<.001$ \\
\hline \multicolumn{7}{|c|}{ GH3 (+1-5); Healthy as anybody else } \\
\hline Con & $2.28[2.22,2.35]$ & $2.25[2.18,2.32]$ & & & & \\
\hline Exp & $2.26[2.21,2.31]$ & $2.23[2.17,2.28]$ & .624 & .448 & 945 & $<.001$ \\
\hline \multicolumn{7}{|c|}{ GH4 $(1-5+)$; Health is getting worse } \\
\hline Con & $3.18[3.13,3.24]$ & $3.30[3.25,3.35]$ & & & & \\
\hline Exp & $2.75[2.71,2.79]$ & $2.81[2.77,2.86]$ & $<.001$ & .012 & .448 & .004 \\
\hline \multicolumn{7}{|c|}{ GH5 (+1-5); Health is excellent } \\
\hline Con & $2.68[2.64,2.71]$ & $2.64[2.61,2.68]$ & & & & \\
\hline Exp & $2.79[2.77,2.82]$ & $2.77[2.74,2.80]$ & $<.001$ & .209 & .773 & .001 \\
\hline
\end{tabular}

Note. $\quad$ Con = control group; Exp = experimental group. Scores are presented as median [95\% confidence interval of median]. 
Effects of Measurement and Group $\times$ Measurement interaction were significant for no item of the RP, BP (Table 3), SF, VT and GH categories of the SF-36 Survey (Table 4).

\section{Discussion}

The current study showed changes in body composition indicated by a decrease in body fat percentage and increase in total muscle mass that followed the yogabased intervention in elderly men. Body fat percentage usually rises in older adults from their 7 th to 8 th decennium followed by its decreasing (Honců et al., 2019; Lacroix, Hortobágyi, Beurskens, \& Granacher, 2017). At the same time, the tendency of muscle mass loss may also be a serious problem in elderly men. According to recent studies (Chatterjee \& Mondal, 2014; Krejci, 2011), our results have suggested that a male organism aged over 65 years, thanks to the testosterone and other anabolic hormones, may respond positively to physical exercises with body fat reduction and increase in muscle mass. The ratio of muscle mass to body fat is significantly influenced by lifestyle, especially food intake, energy expenditure and strength training (Bukova et al., 2019; Ohlsson et al., 2018). The current study suggested that the elderly men probably got into energy balance when their neuromuscular and respiratory systems were stimulated with regular and frequent practicing of yoga exercises. It is also possible that the regular yoga training could support anabolic processes thanks to the presence of testosterone, and as a consequence muscular mass enhanced.

The findings of improvement in static and dynamic balance in elderly men skew in favor of short-term yoga intervention. The English Longitudinal Study of Ageing (Gale et al., 2016) examined many potential factors for incident falls in 1515 men and 1783 women aged $60+$. Besides the age and pain, impairment in static balance indicated by the full-tandem stand was revealed as the most significant mutually adjusted relative risk for men while not for women. Therefore, it seems that the regular practicing of yoga-based exercises can be beneficial to elderly men as it assists in decreasing fall-related incidents. According to the results of the current study, one can recommend male seniors aged $65+$ to develop posture control via yoga exercises, with possible positive effects even after a short time period.

In the current study, the short-term yoga-based intervention resulted in improvement of dynamic balance, too. Dynamic balance, defined as the ability to maintain postural stability and orientation with center of mass over the base of support while the body parts are in motion, (O’Sullivan, Schmitz, \& Fulk, 2014) plays an important role in mobility in the elderly. Dynamic balance was assessed with the use of the Tinetti Balance Assessment Tool that consisted in the observational judgment of features of gait such as hesitation of gait, step length and height, foot clearance, step symmetry and continuity and trunk sway. So, the results of our study suggest that regular practicing of yoga exercises can contribute to optimization of the fundamental locomotion skill such as walking.

However, we consider a bio-psycho-social context to judge changes in posture control and mobility in the elderly. The studies by Dellinger (2017) and Honců et al. (2019) proposed that the maintenance or development of balance ability in the elderly may depend on an interplay of bio-psycho-social factors and determinants. This assumption has been supported by several studies that found positive effects of yoga on stress management, mood harmonization, depression, and anxiety reduction (Pascoe \& Bauer, 2015; Randall, Thomas, Whiting, \& McGrath, 2017; Yoshihara, Hiramoto, Oka, Kubo, \& Sudo, 2014). This is consistent with the findings of a decrease in emotional problems of elderly men in the current study. Specifically, elderly men who regularly practice the yoga-based program perceived reduction of both activity restrictions and concentration problem due to emotional problems (the items RE2 and RE3, Table 4). This finding concurrently with evidence of optimization of postural control suggests that yoga is the appropriate form of regular physical activity for elderly men which can bring significant benefits to their life. This suggestion is consistent with results of the meta-analysis by Youkhana et al. (2016) who reported that yoga may be an optimal form of exercise for older men to improve both physical functioning and mental health, as yoga relieves lower back pain, reduces hypertension, risk of obesity, treats chronic insomnia, and improves mood. Also, Schmid et al. (2010) have indicated that yoga may be a promising intervention to manage the fear of falling and improve balance, thereby reducing fall risk for older adults. One might consider that therapists may explore yoga as a model for balance and falls programming. However, future research is needed to confirm the use of yoga intervention in such programming.

The current study has suggested improvements in the self-care abilities of elderly men following the yoga intervention when they reported decreasing in health limit for activities such as bathing and dressing (the item PF10, Table 3). This finding may be related not only to concurrent optimizing of the balance but also to improved decision-making or mental control. The study by Gothe, Kramer, and McAuley (2014) showed the effect of an 8-week yoga intervention on 
the improvement in executive functions associated with work activity in older adults.

The novelty of the current study consists in the examination of a short-term yoga intervention specifically in elderly men. The yoga program designed for this study included the activities of standing, sitting, and lying on the floor. These activities practiced with a yoga program can stimulate postural control, mobility, and gait speed (Zettergren, Lubeski, \& Viverito, 2011). But it should be noted that the yoga interventions by Zettergren et al. (2011) comprised of the exercises applied only twice or three times per week, whereas participants of the current study performed yoga exercises every day. Each week, they participated in one guided 90-minute training session for one day followed by 10-15 minutes of assisted practice each of the following six days. This type of intervention presents the idea for older people to implement yoga in their daily life.

We believe that short-term yoga interventions are very useful for people aged $65+$. It is necessary to focus on the effects of those interventions in future research in accordance with Sivaramakrishnan et al. (2019). According to Tew et al. (2017), yoga represents a holistic activity with expanding popularity which has the potential to produce a range of physical, mental and social benefits. They declared feasibility and effects of an adapted yoga intervention on physical function and health-related quality of life in physically inactive older adults. With yoga, their chances of doing regularly adequate physical activities without any restrictions and in the required quality of the provided activities would be enhanced. This is in line with the results of the publication outputs of European Commission (2016) and the study by Lacroix et al. (2017).

An important aspect of using short-term yoga interventions is to develop balance and mental health in a spa environment as well, where the usual recommended treatment duration is just 4 weeks (Jandová, Formanová, \& Morávek, 2018; Náprstek, Krejčí, Kajzar, \& Hill, 2019). This represents an entirely new research topic.

\section{Conclusions}

Previous research on the effects of yoga has not involved an examination of effectiveness on a very short yoga-based intervention, specifically on posture control, body composition and mental health in elderly men. Results of this study intimated tendencies for positive change in posture control, functional mobility and body composition after the intensive short-term yoga program in elderly men. To optimize the mental aspects of functioning and health, yoga exercises need to be performed for a longer time period. Further research would be needed to understand what neuromotor and psychic mechanisms may underlie the simultaneous effects of both balance and the mental state during yoga in elderly men.

\section{Acknowledgments}

The study was a part of the project GAČR ID 17-25710S "Basic research of balance changes in seniors" funded by the Czech Science Foundation.

The authors would like to acknowledge and thank all participants and assistants who participated in this study.

\section{Conflict of interest}

There were no conflicts of interest.

\section{References}

Bendíková, E. (2017). Theory of health, movement and lifestyle of human beings. Debrecen, Hungary: University of Debrecen.

Bukova, A., Hagovska, M., Drackova, D., Horbacz, A., Wasik, J., \& Krucanica, L. (2019). Awareness of patients suffering from selected chronic diseases of the importance of physical activity in treating their disorders. Physical Activity Review, 7, 234-239.

Buso, A., Comelli, M., Picco, R., Isola, M., Magnesa, B., Pišot, R., ... Mavelli, I. (2019). Mitochondrial adaptations in elderly and young men skeletal muscle following 2 weeks of bed rest and rehabilitation. Frontiers in Physiology, 10, 474 .

Butterfield, N., Schultz, T., Rasmussen, P., \& Proeve, M. (2017). Yoga and mindfulness for anxiety and depression and the role of mental health professionals: A literature review. Journal of Mental Health Training Education and Practice, 12, 44-54.

Chatterjee, S., \& Mondal, S. (2014). Effect of regular yogic training on growth hormone and dehydroepiandrosterone sulfate as an endocrine marker of aging. Evidence-Based Complementary and Alternatve Medicine, 2014, 240581.

Dellinger, A. (2017). Older adult falls: Effective approaches to prevention. Current Trauma Reports, 3, 118-123.

Ebrahimi, Z., Esmaeilzadeh, G., \& Mohamad, R. (2019). Comparing the efficacy of yoga exercise and intergenerational interaction program on mental health of elderly. Journal of Research \& Health, 9, 401-410.

European Commission. (2016). Senior entrepreneurship: Good practices manual. Retrieved from https://op. europa.eu/en/publication-detail/-/publication/8fdadabd -9ac8-11e6-868c-01aa75ed71a1

Gale, C. R., Cooper, C., \& Aihie Sayer, A. (2016). Prevalence and risk factors for falls in older men and women: 
The English Longitudinal Study of Ageing. Age and Ageing, 45, 789-794.

Gale, C. R., Westbury, L. D., Cooper, C., \& Dennison, E. M. (2018). Risk factors for incident falls in older men and women: The English Longitudinal Study of Ageing. BMC Geriatrics, 18, 117.

Gilleard, C., \& Higgs, P. (2005). Contexts of ageing: Class, cohort and community. Cambridge, United Kingdom: Polity.

Gillespie, L. D., Robertson, M. C., Gillespie, W. J., Sherrington, C., Gates, S., Clemson, L. M., \& Lamb, S. E. (2012). Interventions for preventing falls in older people living in the community. Cochrane Database of Systematic Reviews, 9, CD007146.

Gothe, N. P., Kramer, A. F., \& McAuley, E. (2014). The effects of an 8-week Hatha yoga intervention on executive function in older adults. Journals of Gerontology: Series A, 69, 1109-1116.

Gutenbrunner, C., Ward, A. B., \& Chamberlein, M. A. (2007). White Book on Physical and Rehabilitation Medicine in Europe. Journal of Rehabilitation Medicine, 45(Suppl.), 6-47.

Hafström, A., Malmström, E. M., Terdèn, J., Fransson, P. A., \& Magnusson, M. (2016). Improved balance confidence and stability for elderly after 6 weeks of a multimodal self-administered balance-enhancing exercise program: A randomized single arm crossover study. Gerontology \& Geriatric Medicine, 2, 2333721416644149.

Honců, P., Hill, M., Bičíková, M., Jandová, D., Velíková, M., Kajzar, J., ... Stárka, L. (2019). Activation of adrenal steroidogenesis and an improvement of mood balance in postmenopausal females after spa treatment based on physical activity. International Journal of Molecular Sciences, 20, E3687.

Jandová, D., Formanová, P., \& Morávek, O. (2018). Presenium as a preparing period for senium - benefits of spa stay in the Priessnitz's spa, Ltd. in Jesenik for clients 50+. Acta Salus Vitae, 6(1), 42-50.

Kegelmeyer, D. A., Kloos, A. D., Thomas, K. M., \& Kostyk, S. K. (2007). Reliability and validity of the Tinetti Mobility Test for individuals with Parkinson disease. Physical Therapy, 87, 1369-1378.

Krejci, M. (2011). Yoga training application in overweight control of seniors with arthritis/osteoarthritis. Physiotherapy, 19(2), 3-8.

Krejčí, M. (2019). Intervenční program Život v rovnováze [Intervention program Life in Balance]. In M. Krejčí, V. Hošek, M. Hill, D. Jandová, J. Kajzar, \& P. Bláha (Eds.), Základní výzkum zmén rovnováhy senior (pp. 235-258). Prague, Czech Republic: College of Physical Education and Sport PALESTRA.

Krejci, M., \& Kornatovska, Z. (2017). Yoga applications in persons with disabilities. In E. Bolach \& A. Kawczynski (Eds.), Adaptacyjna aktywnoscz fizyczna (pp. 104-127). Wroclaw, Poland: University School of Physical Education in Wrocław.

Lacroix, A., Hortobágyi, T., Beurskens, R., \& Granacher, U. (2017). Effects of supervised vs. unsupervised training programs on balance and muscle strength in older adults: A systematic review. Sports Medicine, 47, 2341-2361.
Lindahl, E., Tilton, K., Eickholt, N., \& Ferguson-Stegall, L. (2016). Yoga reduces perceived stress and exhaustion levels in healthy elderly individuals. Complementary Therapies in Clinical Practice, 24, 50-56.

Maheshwarananda, P. S. (2000). Yoga in daily life: The system. Vienna, Austria: Ibera Verlag/European University Press.

McHorney, C. A., Ware, J. E., Lu, J. F. R., \& Sherbourne, C. D. (1994). The MOS 36-Item Form Health Survey (SF36): III. Tests of data quality, scaling assumptions and reliability across diverse patient groups. Medical Care, 32(4), 40-66.

Meloun, M., Hill, M., Militký, J., Vrbíková, J., Stanická, S., \& Škrha, J. (2004). New methodology of influential point detection in regression model building for the prediction of metabolic clearance rate of glucose. Clinical Chemistry and Laboratory Medicine, 42, 311-322.

Náprstek, R., Krejčí, M., Kajzar, J., \& Hill, M. (2019). Balance ability improvement of seniors $65+$ during the spa stay. Acta Salus Vitae, 7(2), 65-74.

Ni Chronin, D., Ni Chronin, C., \& Beveridge, A. (2015). Factors influencing deprescribing habits among geriatricians. Age and Ageing, 44, 704-708.

O’Sullivan, S. B., Schmitz, T. J., \& Fulk, G. (2014). Physical rehabilitation (6th ed.). Philadelphia, PA: F. A. Davis Company.

Ohlsson, C., Nethander, M., Karlsson, M. K., Rosengren, B. E., Ribom, E., Mellström, D., \& Vandenput, L. (2018). Serum DHEA and its sulfate are associated with incident fall risk in older men: The MrOS Sweden study. Journal of Bone and Mineral Research, 33, 1227-1232.

Oken, B. S., Zajdel, D., Kishiyama, S., Flegal, K., Dehen, C., Haas, M., ... Leyva, J. (2006). Randomized, controlled, six-month trial of yoga in healthy seniors: Effects on cognition and quality of life. Alternative Therapies in Health and Medicine, 12, 40-47.

Pascoe, M. C., \& Bauer, I. E. (2015). A systematic review of randomised control trials on the effects of yoga on stress measures and mood. Journal of Psychiatric Research, 68, 270-282.

Randall, D., Thomas, M., Whiting, D., \& McGrath, A. (2017). Depression Anxiety Stress Scales (DASS-21): Factor structure in traumatic brain injury rehabilitation. Journal of Head Trauma Rehabilitation, 32, 134-144.

Schmid, A. A., Van Puymbroeck, M., \& Koceja, D. M. (2010). Effect of a 12-week yoga intervention on fear of falling and balance in older adults: A pilot study. Archives of Physical Medicine and Rehabilitation, 91, 576-583.

Shumway-Cook, A., \& Woollacott, M. H. (2016). Motor control: Translating research into clinical practice. Philadelphia, PA: Wolters Kluwer.

Sivaramakrishnan, D., Fitzsimons, C., Kelly, P., Ludwig, K., Mutrie, N., Saunders, D. H., \& Baker, G. (2019). The effects of yoga compared to active and inactive controls on physical function and health related quality of life in older adults - Systematic review and meta-analysis of randomised controlled trials. International Journal of Behavioral Nutrition and Physical Activity, 16, 33.

Tew, G. A., Howsam, J., Hardy, M., \& Bissell, L. (2017). Adapted yoga to improve physical function and health-related quality 
of life in physically-inactive older adults: A randomised controlled pilot trial. BMC Geriatrics, 17, 131.

Tiedemann, A., O'Rourke, S., Sesto, R., \& Sherrington, C. (2013). A 12-week Iyengar yoga program improved balance and mobility in older community-dwelling people: A pilot randomized controlled trial. Journal of Gerontology: Series A, 68, 1068-1075.

Tinetti, M. E., Williams, T. F., \& Mayewski, R. (1986). Fall risk index for elderly patients based on number of chronic disabilities. American Journal of Medicine, 80, 429-434.

Wang, D. (2009). The use of yoga for physical and mental health among older adults: A review of the literature. International Journal of Yoga Therapy, 19, 91-96.

Ware, J. E., Snow, K. K., Kosinski, M., \& Gandek, B. (1993). SF-36 Health Survey: Manual and interpretation guide. Boston, MA: New England Medical Center.
Washington Health Care Association. (2013). Tinetti assessment tool. Retrieved from https://www.whca.org/ files/2013/04/TINETTI_assessment_tool.pdf

Yoshihara, K., Hiramoto, T., Oka, T., Kubo, C., \& Sudo, N. (2014). Effect of 12 weeks of yoga training on the somatization, psychological symptoms, and stress-related biomarkers of healthy women. BioPsychoSocial Medicine, 8, 1. Youkhana, S., Dean, C. M., Wolff, M., Sherrington, C., \& Tiedemann, A. (2016). Yoga-based exercise improves balance and mobility in people aged 60 and over: A systematic review and meta-analysis. Age and Ageing, 45, 21-29.

Zettergren, K. K., Lubeski, J. M., \& Viverito, J. M. (2011). Effects of a yoga program on postural control, mobility, and gait speed in community-living older adults: A pilot study. Journal of Geriatric Physical Therapy, 34, 88-94. 